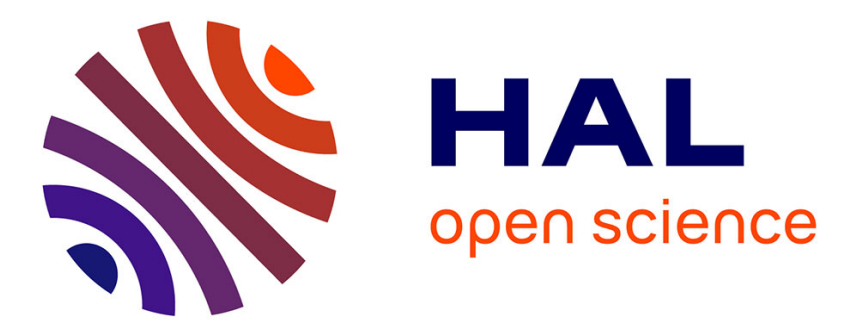

\title{
Fault detection and isolation in linear parameter-varying descriptor systems via proportional integral observer
} Habib Hamdi, Mickael Rodrigues, Chokri Mechmeche, Didier Theilliol, Naceur Benhadj Braiek

\section{- To cite this version:}

Habib Hamdi, Mickael Rodrigues, Chokri Mechmeche, Didier Theilliol, Naceur Benhadj Braiek. Fault detection and isolation in linear parameter-varying descriptor systems via proportional integral observer. International Journal of Adaptive Control and Signal Processing, 2012, 26 (3), pp.224-240. 10.1002/acs.1260 . hal-00605382

\section{HAL Id: hal-00605382 https://hal.science/hal-00605382}

Submitted on 1 Jul 2011

HAL is a multi-disciplinary open access archive for the deposit and dissemination of scientific research documents, whether they are published or not. The documents may come from teaching and research institutions in France or abroad, or from public or private research centers.
L'archive ouverte pluridisciplinaire HAL, est destinée au dépôt et à la diffusion de documents scientifiques de niveau recherche, publiés ou non, émanant des établissements d'enseignement et de recherche français ou étrangers, des laboratoires publics ou privés. 


\title{
Fault Detection and Isolation for Linear Parameter Varying Descriptor Systems via Proportional Integral Observer
}

\author{
H. HAMDI ${ }^{1}$, M. RODRIGUES ${ }^{2}$, C. MECHMECHE ${ }^{1}$, D. THEILLIOL ${ }^{3}$ and \\ N. BENHADJ BRAIEK ${ }^{1}$
}

${ }^{1}$ Laboratoire d'Etude et Commande Automatique des Processus (LECAP), Ecole Polytechnique de Tunisie; (e-mail: hammmdihabib@yahoo.fr,chokri.mechmeche@esstt.rnu.tn,naceur.benhadj@ept.rnu.tn)

${ }^{2}$ Laboratoire d'Automatique et de Génie des Procédés (LAGEP), CNRS UMR 5007, Université Lyon 1, Villeurbanne, F-69622, France (e-mail: rodrigues@lagep.univ-lyon1.fr)

${ }^{3}$ Centre de Recherche en Automatique de Nancy (CRAN), Nancy-Université, CNRS, B.P. 70239, F-54506 Vandouvre-lès-Nancy, FRANCE, (e-mail: didier.theilliol@cran.uhp-nancy.fr)

\section{SUMMARY}

The main contribution of this paper is the design of a polytopic Unknown Input Proportional Integral Observer (UIPIO) for Linear Varying Parameter (LPV) descriptor systems. This observer is used for actuator Fault Detection and Isolation (FDI). The proposed method is based on the representation of LPV descriptor systems in polytopic form where its parameters evolve in a hypercube domain. The designed polytopic UIPIO is also able to estimate the states and the unknown inputs of the LPV descriptor system. Stability conditions of such observer are expressed in terms of Linear Matrix Inequalities (LMI). An example illustrates the performances of such polytopic UIPIO for multiple actuator faults estimation in spite of disturbances.

Copyright (c) 2011 John Wiley \& Sons, Ltd.

Received...

KEY WORDS: LPV descriptor systems, polytopic Proportional Integral Observer, fault detection and isolation, Linear Matrix Inequalities (LMIs)

\section{INTRODUCTION}

The topic of Fault Detection and Isolation (FDI) in dynamic systems has been an active research area in the last two decades, as can be seen, in survey papers by [23] for linear systems, [19] for multi-models representation and [3] for nonlinear systems. For many processes it is feasible to use differential-algebraic equations to describe the plant dynamic behavior. The use of the physical or chemical laws is often a natural choice. In the most general form, a set of so-called descriptor system equations can be used as discussed by [4].

Model-based FDI techniques use mathematical models of the monitored process and extract features from measured signals, to generate fault indicating signals, that is, the residuals. Hence, generation of residual signals is a central issue in model-based FDI and a rich variety of methods are available for FDI. The basic idea behind the observer-based approaches is to estimate the outputs of the system from the measurements by using observers. Then, the output estimation error is used as a residual.

The problem of designing observers has been investigated by many authors. The authors in [8] have proposed full and reduced order observers for linear descriptor systems. In [11], the authors have developed an observer design method based on singular value decompositions and the concept of generalized inverse matrix. Observer design for nonlinear descriptor systems was studied in [4]. The authors in [14] have proposed an unknown inputs multi-observer for nonlinear descriptor systems 
described by multi-models. As in the conventional systems, in view of the advantages of integral actions, some researchers have introduced the integral term in observer design for descriptor systems $[1,13]$. Full and reduced order Proportional Integral (PI) observers for fuzzy descriptor system subject to parameter variations have been designed in [12]. These observers allow estimation of both the state and the unknown inputs. The authors in [24], [22] have synthesized robust control laws for fuzzy descriptor systems. More recently, Linear Parameter Varying (LPV) modeling of the monitored system has been considered. Such models can be used efficiently to represent some ordinary nonlinear systems. Some researchers from the fault detection and isolation community have developed model-based FDI methods [9, 26] and Fault Tolerant Control [20] using LPV systems. For polytopic LPV systems, a polytopic unknown inputs observer has been also used for the state estimation of uncertain systems and for FDI of nonlinear systems described by a multi-models representation [21].

The authors in $[16,17]$ have designed gain-scheduled controllers for LPV descriptor systems. In spite of that, there are few studies on state reconstruction and fault diagnosis for LPV descriptor systems despite of a method for actuator fault detection and estimation proposed in [10].

The main contribution of this paper is associated with the design of a polytopic Unknown Input Proportional Integral Observer (UIPIO) for LPV descriptor systems and for fault detection and isolation. In this modeling framework, the dynamic behavior of the LPV descriptor system is given in a polytopic form, that allows to describe the system as a convex combination of subsystems defined by the vertices of a convex polytope. These submodels are then combined by a convex weighing functions to yield the global model.

The first step of this work consists of modeling the LPV descriptor system by a polytopic form. The second step is devoted to the design of a polytopic UIPIO for LPV descriptor systems subjected to external disturbances. The obtained observer is used for state, unknown inputs estimation and for actuator faults detection and isolation. Note that, in one hand, to estimate the state and unknown inputs, all matrices of the LPV descriptor systems are considered with time-varying parameters. On the other hand, to ensure the fault detection and estimation, the control matrix and disturbance matrix of the dynamic systems are considered with static parameters.

This paper is organized as follows: The polytopic representation of LPV descriptor systems is presented in Section 2. The polytopic Unknown Input Proportional Integral Observer (UIPIO) design corresponding to the polytopic models and its stability conditions are studied in Section 3. Section 4 is devoted to detection, isolation and estimation of the actuator faults by using a set of polytopic UIPIO for LPV descriptor systems. Finally, an illustrative example is provided to show the effectiveness of the proposed approach.

In this paper, the notation is standard. $\mathbb{R}$ denotes the set of real numbers; $\mathcal{C}$ is the complex plane; $A^{+}$denotes the generalized inverse of $A ; Q>0$ or $(Q<0)$ indicates that the matrix $Q$ is positive (or negative) definite. Also, $\forall$ means "for all".

\section{POLYTOPIC LPV DESCRIPTOR SYSTEM}

Consider the class of LPV descriptor systems:

$$
\left\{\begin{array}{l}
E \dot{x}(t)=\widetilde{A}(\theta(t)) x(t)+\widetilde{B}(\theta(t)) u(t)+\widetilde{R}(\theta(t)) d(t) \\
y(t)=C x(t)
\end{array}\right.
$$

$x(t) \in \mathbb{R}^{n}$ is the state vector, $u(t) \in \mathbb{R}^{p}$ is the control input, $d(t) \in \mathbb{R}^{q}$ is the unknown input vector and $y(t) \in \mathbb{R}^{m}$ represents the measured output vector. $\widetilde{A}(\cdot), \widetilde{B}(\cdot), \widetilde{R}(\cdot)$ are continuous functions which depend affinely on the time-varying parameter vector $\theta(t) \in \mathbb{R}^{l}$. It is also assumed that this time-varying parameter vector $\theta(t)$ is bounded and lies into a hypercube such that $[2,6,25]$ :

$$
\theta(t) \in \Gamma=\left\{\theta \mid \underline{\theta}_{i}(t) \leq \theta(t) \leq \bar{\theta}_{i}(t)\right\}, \quad \forall t \geq 0
$$


The LPV descriptor system (1) with the affine parameter dependence (2) allows us to determine a set of matrices $\Pi_{i}$ such that:

$$
\Pi(\theta(t))=\Pi_{0}+\sum_{i=1}^{h} \theta_{i}(t) \Pi_{i}, \quad \forall \theta(t) \in \Gamma
$$

where

$$
\Pi=\left(\begin{array}{ccc}
\widetilde{A} & \widetilde{B} & \widetilde{R} \\
C & 0 & 0
\end{array}\right)
$$

The system (1) can be transformed into a convex interpolation of the vertices of $\Gamma$ where the vertices of the polytope are defined such that [20]: $S_{i}=\left[A_{i}, B_{i}, C, R_{i}\right], \forall i \in[1, \ldots, h]$ where $h=2^{l}$. The polytopic coordinates are denoted $\rho(\theta(t))$ and vary into the convex set $\Omega$ :

$$
\Omega=\left\{\rho(\theta(t)) \in \mathbb{R}^{h}, \rho(\theta(t))=\left[\rho_{1}(\theta(t)), \ldots, \rho_{h}(\theta(t)]^{T}, \rho_{i}(\theta(t)) \geq 0, \sum_{i=1}^{h} \rho_{i}(\theta(t))=1\right\}\right.
$$

Consequently, the polytopic LPV descriptor system with the time-varying parameter vector $\rho(\theta(t)) \in \Omega$ is given by:

$$
\left\{\begin{array}{l}
E \dot{x}(t)=\sum_{i=1}^{h} \rho_{i}(\theta(t))\left(A_{i} x(t)+B_{i} u(t)+R_{i} d(t)\right) \\
y(t)=C x(t)
\end{array}\right.
$$

where $A_{i} \in \mathbb{R}^{n \times n}, B_{i} \in \mathbb{R}^{n \times p}, R_{i} \in \mathbb{R}^{n \times q}$ and $C \in \mathbb{R}^{m \times n}$ are time invariant matrices defined for the $i^{\text {th }}$ model. $E \in \mathbb{R}^{\text {nxn }}$ is a constant matrix and $\operatorname{rank}(E)=r<n$.

The following section presents a design method of a polytopic Unknown Input Proportional Integral Observer (UIPIO) for LPV descriptor systems subjects to disturbances. This observer is constructed by the interpolation of a set of sub-UIPIO, according to the parameter $\rho_{i}(\theta(t))$. As in ordinary multi-models representation [19] and for multi-observer design, the observability requires that all the subsystems are observable. Then, the following assumptions express the observability properties of the polytopic LPV descriptor system.

Assumption A1: The matrix $C$ is of full-row rank, i.e., $\operatorname{rank}(C)=m$.

Assumption A2: The triple matrix $\left(E, A_{i}, C\right)$ is R-observable [12], $\forall i=1, \ldots, h$, i.e.,

$$
\operatorname{rank}\left[\begin{array}{c}
s E-A_{i} \\
C
\end{array}\right]=n, \forall s \in \mathcal{C} .
$$

Assumption A3: The triple matrix $\left(E, A_{i}, C\right)$ is impulse-observable [12], $\forall i=1, \ldots, h$, i.e.,

$$
\operatorname{rank}\left[\begin{array}{cc}
E & A_{i} \\
0 & E \\
0 & C
\end{array}\right]=n+\operatorname{rank}(E)
$$

Descriptor system is represented by a combined set of Differential and Algebraic Equations (DAE). The observability reflects the capacity to estimate the whole state. Then, two types of observability are required:

The R-observability characterizes the capacity to reconstruct only the state of the dynamic part (governed by differential equations).

The impulse observability guarantees the capacity to estimate the state of static part (governed by algebraic equations). 


\section{POLYTOPIC UNKNOWN INPUT PROPORTIONAL INTEGRAL OBSERVER DESIGN}

Polytopic UIPIO of LPV descriptor system (6) is described by the following equations:

$$
\left\{\begin{array}{l}
\dot{z}(t)=\sum_{i=1}^{h} \rho_{i}(\theta(t))\left(N_{i} z(t)+G_{i} u(t)+L_{i} y(t)+T_{i} \hat{d}(t)\right) \\
\hat{x}(t)=z(t)+H_{2} y(t) \\
\dot{\hat{d}}(t)=\sum_{i=1}^{h} \rho_{i}(\theta(t)) \Phi_{i}(y(t)-\hat{y}(t))
\end{array}\right.
$$

where $\hat{x}(t) \in \mathbb{R}^{n}, z(t) \in \mathbb{R}^{n}$ and $\hat{d}(t) \in \mathbb{R}^{q}$ are the estimated state vector, transformed state vector and estimated unknown inputs vector respectively. $N_{i}, G_{i}, L_{i}, T_{i}, H_{2}$ and $\Phi_{i}$ are unknown matrices of appropriate dimensions. The unknown inputs can be regarded as signals to be estimated. This polytopic UIPIO (9) aggregates all local observers defined at each vertex.

\section{Definition 1}

System (9) is called a polytopic UIPIO for system (6) if for arbitrary initial conditions $x(0)$ and $z(0)$ and arbitrary input $u(t)$, the following relations hold:

$$
\begin{aligned}
& \lim _{t \rightarrow \infty}(x(t)-\hat{x}(t))=0 \\
& \lim _{t \rightarrow \infty}(d(t)-\hat{d}(t))=0, \quad \forall \hat{d}(0)
\end{aligned}
$$

The state estimation error is equal to: $e(t)=x(t)-\hat{x}(t)$; then it follows from (6) and (9) that

$$
\begin{aligned}
& e(t)=x(t)-z(t)-H_{2} C x(t) \\
& e(t)=\left(I_{n}-H_{2} C\right) x(t)-z(t)
\end{aligned}
$$

Assuming there exists a matrix $H_{1} \in R^{n \times n}$ such that:

$$
H_{1} E=I_{n}-H_{2} C
$$

which is equivalent to

$$
\left[\begin{array}{ll}
H_{1} & H_{2}
\end{array}\right]\left[\begin{array}{l}
E \\
C
\end{array}\right]=I_{n}
$$

Equation (13) has a solution if $\operatorname{rank}\left[\begin{array}{l}E \\ C\end{array}\right]=n$. By using (12), the state estimation error becomes:

$$
e(t)=H_{1} E x(t)-z(t)
$$

Now, we assume that the bounded unknown inputs are defined with slow variation, i.e $\dot{d}(t) \simeq 0$. Then, for $\zeta(t)=d(t)-\hat{d}(t)$ the derivative of the unknown input is

$$
\dot{\zeta}(t)=-\dot{\hat{d}}(t)
$$

By taking into account (6), (9) and (14), the dynamics of the estimation error is given by the following equation:

$$
\dot{e}(t)=H_{1} E \dot{x}(t)-\dot{z}(t)
$$

$\dot{e}(t)=\sum_{i=1}^{h} \rho_{i}(\theta(t))\left(N_{i} e(t)+\left(H_{1} A_{i}-L_{i} C-N_{i} H_{1} E\right) x(t)+\left(H_{1} B_{i}-G_{i}\right) u(t)+T_{i} \zeta(t)+\left(H_{1} R_{i}-T_{i}\right) d(t)\right)$ 
If the following relations hold true $\forall i=1, \ldots, h$ :

$$
\begin{aligned}
& H_{1} A_{i}-N_{i} H_{1} E-L_{i} C=0 \\
& G_{i}=H_{1} B_{i} \\
& H_{1} E=I_{n}-H_{2} C \\
& T_{i}=H_{1} R_{i}
\end{aligned}
$$

and from (9), (15) and (17), the dynamics of the state estimation and the unknown input errors become:

$$
\begin{aligned}
& \dot{e}(t)=\sum_{i=1}^{h} \rho_{i}(\theta(t))\left(N_{i} e(t)+T_{i} \zeta(t)\right) \\
& \dot{\zeta}(t)=-\sum_{i=1}^{h} \rho_{i}(\theta(t))\left(\Phi_{i} C(x(t)-\hat{x}(t))\right) \\
& \dot{\zeta}(t)=-\sum_{i=1}^{h} \rho_{i}(\theta(t))\left(\Phi_{i} C\right) e(t)
\end{aligned}
$$

The above errors dynamics can be represented by connecting (24) to (22) as follows:

$$
\left[\begin{array}{c}
\dot{e}(t) \\
\dot{\zeta}(t)
\end{array}\right]=\sum_{i=1}^{h} \rho_{i}(\theta(t))\left[\begin{array}{cc}
N_{i} & T_{i} \\
-\Phi_{i} C & 0
\end{array}\right]\left[\begin{array}{c}
e(t) \\
\zeta(t)
\end{array}\right]
$$

If the above augmented error dynamic equation is stable, the state estimation will converge asymptotically to the real state.

\subsection{UIPIO Matrices Computing}

In order to find matrix [ $H_{1} H_{2}$ ], it follows that equation (13) has a solution since

$\operatorname{rank}\left[\begin{array}{c}E \\ C\end{array}\right]=n$, and the solution is given by

$$
\left[\begin{array}{ll}
H_{1} & H_{2}
\end{array}\right]=\left[\begin{array}{l}
E \\
C
\end{array}\right]^{+}
$$

By substituting (20) into (18), we obtain $\forall i=1, \ldots, h$ :

$$
H_{1} A_{i}=N_{i}\left(I_{n}-H_{2} C\right)+L_{i} C
$$

Equation (27) can be equivalently written as:

$$
N_{i}=H_{1} A_{i}-K_{i} C
$$

with

$$
K_{i}=L_{i}-N_{i} H_{2}
$$

and matrices $L_{i}$ can be deduced from equation (29) as follows:

$$
L_{i}=K_{i}+N_{i} H_{2}
$$

To compute the gains $K_{i}$ and $\Phi_{i}$, the estimated errors equation (25) can be written as:

$$
\left[\begin{array}{l}
\dot{e}(t) \\
\dot{\zeta}(t)
\end{array}\right]=\sum_{i=1}^{h} \rho_{i}(\theta(t))\left(\bar{A}_{i}-\bar{K}_{i} \bar{C}\right)\left[\begin{array}{l}
e(t) \\
\zeta(t)
\end{array}\right]
$$


or equivalently such that:

$$
\dot{e}_{a}(t)=\sum_{i=1}^{h} \rho_{i}(\theta(t))\left(\bar{A}_{i}-\bar{K}_{i} \bar{C}\right) e_{a}(t)=\mathcal{A}(t) e_{a}(t)
$$

$\bar{A}_{i}=\left[\begin{array}{cc}H_{1} A_{i} & T_{i} \\ 0 & 0\end{array}\right], \bar{K}_{i}=\left[\begin{array}{c}K_{i} \\ \Phi_{i}\end{array}\right], \bar{C}=\left[\begin{array}{ll}C & 0\end{array}\right], e_{a}(t)=\left[\begin{array}{c}e(t) \\ \zeta(t)\end{array}\right]$ and $T_{i}=H_{1} R_{i}$.

Note that this estimation error (32) is a continuous function which depends on the time-varying parameter vector $\rho(t) \in \Omega$.

Obviously, the stability of (32) is ensured if and only if the pair $\left(\bar{A}_{i}, \bar{C}\right)$ is detectable $\forall i=1, \ldots, h$ which is equivalent to:

$$
\operatorname{rank}\left[\begin{array}{cc}
s E-H_{1} A_{i} & -H_{1} R_{i} \\
0 & s I_{q} \\
C & 0
\end{array}\right]=n+q, \quad \forall s \in \mathcal{C}^{+} .
$$

Equation (33) is the generalization of R-detectability [12] for the polytopic descriptor system.

\subsection{Stability and convergence conditions}

The exponential convergence of the estimation error ( $\alpha$-stability of the observer) is a way to ensure a convergence velocity of the estimation error via a decay rate and to improve dynamic performances of the observer [18]. The following theorem provides sufficient conditions for ensuring the exponential convergence of the estimation error (32).

\section{Theorem 1}

Let consider the system (6) and the polytopic UIPIO (9). The exponential convergence of the estimation error (32) is guaranteed if there exists a symmetric and positive definite matrix $Q$, matrices $W_{i}=Q \bar{K}_{i}$ and a positive scalar $\alpha$ for all $\rho \in \Omega$ such that:

$$
\bar{A}_{i}^{T} Q+Q \bar{A}_{i}-\bar{C}^{T} W_{i}^{T}-W_{i} \bar{C}+2 \alpha Q<0, \quad \forall i \in[1, \ldots, h]
$$

\section{Proof 1}

The proof of this theorem is performed by using the following quadratic Lyapunov function:

$$
V(t)=e_{a}^{T}(t) Q e_{a}(t), \quad Q=Q^{T}>0
$$

The exponential convergence of the estimation error is guaranteed if:

$$
\exists Q=Q^{T}>0: \quad \dot{V}(t)+2 \alpha V(t)<0
$$

where $\alpha$ is called the decay rate. Indeed, the solution of the Equation (36) is given by:

$$
V(t) \leq V(0) \exp (-2 \alpha t), \forall t \geq 0
$$

Due to $\lambda_{\min }(Q)\left\|e_{a}(t)\right\|^{2} \leq V(t) \leq \lambda_{\max }(Q)\left\|e_{a}(t)\right\|^{2}$, the norm of the estimation error can be bounded by:

$$
\left\|e_{a}(t)\right\| \leq \sqrt{\frac{\lambda_{\max }(Q)}{\lambda_{\min }(Q)}} \exp (-\alpha t)\left\|e_{a}(0)\right\|, \forall t \geq 0
$$

The derivative of (35) with respect to time yields:

$$
\dot{V}(t)=\dot{e}_{a}^{T} Q e_{a}+e_{a}^{T}(t) Q \dot{e}_{a}(t)
$$


that becomes by using equation (32):

$$
\dot{V}(t)=e_{a}(t)^{T}\left\{\mathcal{A}(t)^{T} Q+Q \mathcal{A}(t)\right\} e_{a}(t)
$$

By using (35) and (40), the inequality (36) becomes:

$$
e_{a}(t)^{T}\left\{\mathcal{A}(t)^{T} Q+Q \mathcal{A}(t)+2 \alpha Q\right\} e_{a}(t)
$$

that is a quadratic form in $e_{a}(t)$. Therefore, the above inequality is satisfied if the following inequality holds:

$$
\mathcal{A}(t)^{T} Q+Q \mathcal{A}(t)+2 \alpha Q<0
$$

that is a sufficient condition for ensuring the exponential convergence of the estimation error $e_{a}(t)$. By considering the fact that $Q$ is positive definite matrix and that $\mathcal{A}(t)$ is a continuous function of parameter $\rho \in \Omega$, and $\Omega$ is compact: it is clear that if there exists a positive definite matrix $Q$ for all $\rho \in \Omega$ it implies that the left hand side of equation (42) is uniformly negative definite [25]. Then, from equation (32), let replace $\mathcal{A}(t)$ by $\sum_{i=1}^{h} \rho_{i}(\theta(t))\left(\bar{A}_{i}-\bar{K}_{i} \bar{C}\right)=\sum_{i=1}^{h} \rho_{i}(\theta(t)) \Psi_{i}$. Hence, the inequality (42) becomes for all $\rho_{i}(\theta(t)) \in \Omega$ :

$$
\sum_{i=1}^{h} \rho_{i}(\theta(t)) \Psi_{i}^{T} Q+Q \sum_{i=1}^{h} \rho_{i}(\theta(t)) \Psi_{i}+2 \alpha Q<0
$$

For any $\rho_{i}(\theta(t)) \in \Omega$ and due to the properties of this compact set [15], the previous inequality (43) becomes

$$
\sum_{i=1}^{h} \rho_{i}(\theta(t)) \Psi_{i}^{T} Q+Q \sum_{i=1}^{h} \rho_{i}(\theta(t)) \Psi_{i}+\sum_{i=1}^{h} \rho_{i}(\theta(t)) 2 \alpha Q<0
$$

The inequality (44) holds if the following inequalities are satisfied over all $\rho_{i}(\theta(t)) \in \Omega$ :

$$
\Psi_{i}^{T} Q+Q \Psi_{i}+2 \alpha Q<0, \quad \forall i \in[1, \ldots, h]
$$

or equivalently by substituting $\Psi_{i}$ :

$$
\bar{A}_{i}^{T} Q+Q \bar{A}_{i}-Q \bar{K}_{i} \bar{C}-\left(\bar{K}_{i} \bar{C}\right)^{T} Q+2 \alpha Q<0, \forall i \in[1, \ldots, h]
$$

Let us notice that the above inequalities are bilinear in $\bar{K}_{i}$ and $Q$. Therefore, it is not possible to solve them directly using standard convex optimization algorithms. However, the following change of variable: $W_{i}=Q \bar{K}_{i}$ can be useful in order to linearize (46) which becomes:

$$
\bar{A}_{i}^{T} Q+Q \bar{A}_{i}-\bar{C}^{T} W_{i}^{T}-W_{i} \bar{C}+2 \alpha Q<0, \forall i \in[1, \ldots, h]
$$

that are linear inequalities in $Q$ and $W_{i}$. Now, a solution can be found using classical LMI tools and the gains are computed with $\bar{K}_{i}=Q^{-1} W_{i}, \forall i \in[1, \ldots, h]$.

Remark : Exponential convergence of estimation error (32) is a strong form of convergence; it implies asymptotic convergence [25]. Indeed, the asymptotic convergence of the estimation error is obtained by considering a decay rate equal to zero $(\alpha=0)$.

In the following, the proposed observer is used for fault detection, isolation and estimation of actuator fault with disturbance rejection. 


\section{FAULT DETECTION AND ISOLATION IN LPV DESCRIPTOR SYSTEM}

Fault diagnosis is usually performed to accomplish one or more of the following tasks: fault detection (or monitoring), to indicate the fault occurrence; fault isolation, to determine the location of the fault and fault identification, to estimate the fault magnitude.

However, the relative importance of these three tasks are obviously subjective even if fault detection and isolation are an absolute must for any practical system. Fault identification (estimation), which is certainly helpful, may not be essential if no reconfiguration action is involved. Hence, fault diagnosis is very often considered as fault detection and isolation.

In this section, the polytopic UIPIO are specifically designed for fault detection, isolation and estimation. In the framework of robust fault detection, we are looking forward to generate residual signals that indicate if a fault occurs or not.

\subsection{Residual generation via polytopic UIPIO}

A residual generation algorithm using a polytopic UIPIO is considered in order to be sensitive to an actuator fault vector $f \in R^{f}$ and insensitive to the disturbance vector $d(t)$ [23].

Consider the following polytopic descriptor system with a constant control matrix $B_{i}$, i.e. $B_{i}=B$ and a constant unknown input matrix $R_{i}=R$ :

$$
\left\{\begin{array}{l}
E \dot{x}(t)=\sum_{i=1}^{h} \rho_{i}(\theta(t))\left(A_{i} x(t)+B u(t)+R d(t)+F f(t)\right) \\
y(t)=C x(t)
\end{array}\right.
$$

For residual generation purposes of (48), the polytopic UIPIO has the following form:

$$
\left\{\begin{array}{l}
\dot{z}(t)=\sum_{i=1}^{h} \rho_{i}(\theta(t))\left(N_{i} z(t)+G_{i} u(t)+L_{i} y(t)+T \hat{f}(t)\right) \\
\hat{x}(t)=z(t)+H_{2} y(t) \\
r(t)=y(t)-\hat{y}(t) \\
\dot{\hat{f}}(t)=\sum_{i=1}^{h} \rho_{i}(\theta(t)) \Phi_{i}(y(t)-\hat{y}(t))
\end{array}\right.
$$

where $r(t)$ is the so-called residual signal generated by the comparison between measured and estimated outputs. The diverse matrices in (49) will be determined to ensure the convergence of the estimated errors. In order to describe the dynamic of polytopic UIPIO (49), the estimation error and the residual equations are defined by: $e(t)=x(t)-\hat{x}(t)$ and $r(t)=C e(t)$. It can be shown that:

$\dot{e}(t)=\sum_{i=1}^{h} \rho_{i}(\theta(t)) N_{i} e(t)+\left(H_{1} A_{i}-N_{i} H_{1} E-L_{i} C\right) x(t)+\left(H_{1} B-G\right) u(t)+H_{1} R d(t)+\left(H_{1} F-T\right) f(t)+T \zeta_{f}(t)$

where

$$
\zeta_{f}(t)=f(t)-\hat{f}(t)
$$

The following conditions have to be verified in order to provide robustness to unknown inputs and sensitivity to faults. Then, equations (18) to (20) and the following equations must be satisfied:

$$
\begin{aligned}
& T=H_{1} F \\
& H_{1} R=0
\end{aligned}
$$

Hence, equation (50) becomes:

$$
\dot{e}(t)=\sum_{i=1}^{h} \rho_{i}(\theta(t))\left(N_{i} e(t)+T \zeta_{f}(t)\right)
$$


By assuming the fault as a step, we find:

$$
\dot{\zeta}_{f}(t)=-\dot{\hat{f}}(t)
$$

Thus, from (49) we obtain:

$$
\dot{\hat{f}}(t)=-\sum_{i=1}^{h} \rho_{i}(\theta(t)) \Phi_{i} C e(t)
$$

Equations (54) and (56) can be combined in an augmented form as follows:

$$
\left[\begin{array}{c}
\dot{e}(t) \\
\dot{\zeta}_{f}(t)
\end{array}\right]=\sum_{i=1}^{h} \rho_{i}(\theta(t))\left[\begin{array}{cc}
N_{i} & T \\
-\Phi_{i} C & 0
\end{array}\right]\left[\begin{array}{c}
e(t) \\
\zeta_{f}(t)
\end{array}\right]
$$

The design of the polytopic UIPIO (49) is equivalent to solve equations (18) to (20) under constraint (53) and to ensure that the augmented error equation (57) is stable. Then, matrices $H_{1}$ and $H_{2}$ are determined such that constraints (20) and (53) are satisfied. Equations (20) and (53) are rewritten in an augmented matrix equation as

$$
\left[\begin{array}{ll}
H_{1} & H_{2}
\end{array}\right]\left[\begin{array}{cc}
E & R \\
C & 0
\end{array}\right]=\left[\begin{array}{ll}
I_{n} & 0
\end{array}\right]
$$

A solution of (58) exists if [13]:

$$
\operatorname{rank}\left[\begin{array}{cc}
E & R \\
C & 0
\end{array}\right]=n+\operatorname{rank}(R)
$$

Under (59), the general solution of (58) is:

$$
\left[\begin{array}{ll}
H_{1} & H_{2}
\end{array}\right]=\left[\begin{array}{ll}
I_{n} & 0
\end{array}\right]\left[\begin{array}{cc}
E & R \\
C & 0
\end{array}\right]^{+}
$$

The remaining matrices $G_{i}, N_{i}$ and $L_{i}$ of this polytopic UIPIO based residual generator are given by (19), (28) and (30) respectively.

The residuals are generated by integrating the estimated output errors and the occurence of faults are detected by its magnitudes. Thus, it is verified that the polytopic UIPIO based residual generator can estimate the state variables and detect faults at the same time.

\subsection{Actuator Fault Isolation}

The fault isolation problem is to determine in which actuator the fault has occurred. The idea is to make each residual sensitive to only one actuator fault and insensitive to all other faults [19]. To design robust actuator fault isolation scheme, only one component of $u(t)$ is considered faulty. The others are assumed to be fault free and considered as unknown inputs. Hence, for each actuator fault, the polytopic LPV descriptor system corrupted by faults and unknown inputs is described by:

$$
\left\{\begin{array}{l}
E \dot{x}(t)=\sum_{i=1}^{h} \rho_{i}(\theta(t))\left(A_{i} x(t)+B u(t)+\left[\begin{array}{ll}
R & F^{r}
\end{array}\right]\left[\begin{array}{c}
d(t) \\
f_{d}(t)
\end{array}\right]+F^{j} f_{j}(t)\right) \\
y(t)=C x(t)
\end{array}\right.
$$

where $F^{j}$ is the $j^{t h}$ column of $B$ with $j \in[1, \ldots, p], F^{r}$ is a matrix of the remaining columns of $B . f_{j}$ represents the faults to be isolated and estimated and $f_{d}(t)$ is considered in this FDI scheme as an unknown input. [ $\left.R \begin{array}{cc}R & F^{r}\end{array}\right]$ is the new disturbance matrix composed by the original unknown inputs matrix $R$ and the matrix $F_{r}$. Based on the above system description, $p$ polytopic UIPIO based 
residual generators can be synthesized for $j \in[1, \ldots, p]$ such that:

$$
\left\{\begin{aligned}
\dot{z}^{j}(t) & =\sum_{i=1}^{h} \rho_{i}(\theta(t))\left(N_{i}^{j} z(t)+G_{i}^{j} u(t)+L_{i}^{j} y(t)+T \hat{f}_{j}(t)\right) \\
r^{j}(t) & =y(t)-\hat{y}(t) \\
\dot{\hat{f}}_{j}(t) & =\sum_{i=1}^{h} \rho_{i}(\theta(t)) \Phi_{i}^{j}(y(t)-\hat{y}(t))
\end{aligned}\right.
$$

The observer matrices must satisfy the equations (19), (28), (29) and (60) such that the initial matrix $R$ is replaced by $\left[\begin{array}{ll}R & F^{r}\end{array}\right]$. Each residual vector $r^{j}(t)$ produced by the $j^{t h}$ polytopic UIPIO, may be used to isolate a fault according to a statistical test [21]. The isolation scheme can only isolate a single fault at the same time. This is based on the fact that the probability for two or more faults to occur at the same time is very small in a real situation.

\section{ILLUSTRATIVE EXAMPLE}

Let us consider the following LPV descriptor system defined by

$$
\left\{\begin{array}{l}
E \dot{x}(t)=A(\theta(t)) x(t)+B(\theta(t)) u(t)+R(\theta(t)) d(t) \\
y(t)=C x(t)
\end{array}\right.
$$

where

$$
\begin{gathered}
A(\theta)=\left[\begin{array}{cccc}
-1.75+\theta_{2} & 1 & 0 & 0 \\
-1 & -1+\theta_{1} & 0 & 0 \\
-1.8 & -1 & -0.75+\theta_{1} & 0 \\
-1 & 0 & 0 & -1+\theta_{2}
\end{array}\right], E=\left[\begin{array}{llll}
1 & 0 & 0 & 0 \\
0 & 1 & 0 & 0 \\
0 & 0 & 0 & 0 \\
0 & 0 & 0 & 0
\end{array}\right] \\
B(\theta)=\left[\begin{array}{cc}
1+\theta_{1} & 1 \\
1 & 0.5+\theta_{2} \\
1 & 0 \\
\theta_{2} & 0
\end{array}\right], R(\theta)=\left[\begin{array}{c}
0 \\
0.6+\theta_{1} \\
0 \\
1
\end{array}\right] \text { and } C=\left[\begin{array}{llll}
1 & 0 & 1 & 0 \\
0 & 1 & 0 & 1 \\
0 & 0 & 1 & 1
\end{array}\right]
\end{gathered}
$$

In this example, the polytopic LPV descriptor system is considered where variables $\theta_{i}$ vary according to $\theta_{1} \in[-0.05,0.05]$ and $\theta_{2} \in[-0.1,0.1], l=2$. In this case, the polytopic representation can be written as follows:

$$
\left\{\begin{array}{l}
E \dot{x}(t)=\sum_{i=1}^{4} \rho_{i}(\theta(t))\left(A_{i} x(t)+B_{i} u(t)+R_{i} d(t)\right) \\
y(t)=C x(t)
\end{array}\right.
$$

The obtained system evolves in a polytope of 4 vertices corresponding to the extreme values of the parameters $\theta_{1}$ and $\theta_{2}$. The system matrices are given:

$$
\begin{aligned}
& A_{1}=\left[\begin{array}{cccc}
-1.85 & 1 & 0 & 0 \\
-1 & -1.05 & 0 & 0 \\
-1.8 & -1 & -0.8 & 0 \\
-1 & 0 & 0 & -1.1
\end{array}\right], A_{2}=\left[\begin{array}{cccc}
-1.65 & 1 & 0 & 0 \\
-1 & -1.05 & 0 & 0 \\
-1.8 & -1 & -0.8 & 0 \\
-1 & 0 & 0 & -0.9
\end{array}\right] \\
& A_{3}=\left[\begin{array}{cccc}
-1.85 & 1 & 0 & 0 \\
-1 & -0.95 & 0 & 0 \\
-1.8 & -1 & -0.7 & 0 \\
-1 & 0 & 0 & -1.1
\end{array}\right], A_{4}=\left[\begin{array}{cccc}
-1.65 & 1 & 0 & 0 \\
-1 & -0.95 & 0 & 0 \\
-1.8 & -1 & -0.7 & 0 \\
-1 & 0 & 0 & -0.9
\end{array}\right] \\
& B_{1}=\left[\begin{array}{cc}
0.95 & 1 \\
1 & 0.4 \\
1 & 0 \\
-0.1 & 0
\end{array}\right], B_{2}=\left[\begin{array}{cc}
0.95 & 1 \\
1 & 0.6 \\
1 & 0 \\
0.1 & 0
\end{array}\right], B_{3}=\left[\begin{array}{cc}
1.05 & 1 \\
1 & 0.4 \\
1 & 0 \\
-0.1 & 0
\end{array}\right], B_{4}=\left[\begin{array}{cc}
1.05 & 1 \\
1 & 0.6 \\
0 & 0 \\
0.1 & 0
\end{array}\right]
\end{aligned}
$$




$$
R_{1}=R_{2}=\left[\begin{array}{c}
0 \\
0.55 \\
0 \\
1
\end{array}\right], R_{3}=R_{4}=\left[\begin{array}{c}
0 \\
0.65 \\
0 \\
1
\end{array}\right] \text { and } C=\left[\begin{array}{cccc}
1 & 0 & 1 & 0 \\
0 & 1 & 0 & 1 \\
0 & 0 & 1 & 1
\end{array}\right]
$$

The weighing functions $\rho_{i}(\theta(t))$ are defined such that:

$$
\begin{aligned}
& \rho_{1}(\theta(t))=\frac{\theta_{1}-\underline{\theta}_{1}}{\bar{\theta}_{1}-\underline{\theta}_{1}} \frac{\theta_{2}-\underline{\theta}_{2}}{\bar{\theta}_{2}-\underline{\theta}_{2}}=\frac{\left(\theta_{1}+0.05\right)\left(\theta_{2}+0.1\right)}{0.02}, \rho_{2}(\theta(t))=\frac{\theta_{1}-\underline{\theta}_{1}}{\bar{\theta}_{1}-\underline{\theta}_{1}} \frac{\bar{\theta}_{2}-\theta_{2}}{\bar{\theta}_{2}-\underline{\theta}_{2}}=\frac{\left(\theta_{1}+0.05\right)\left(0.1-\theta_{2}\right)}{0.02} \\
& \rho_{3}(\theta(t))=\frac{\bar{\theta}_{1}-\theta_{1}}{\bar{\theta}_{1}-\underline{\theta}_{1}} \frac{\theta_{2}-\underline{\theta}_{2}}{\bar{\theta}_{2}-\underline{\theta}_{2}}=\frac{\left(0.05-\theta_{1}\right)\left(\theta_{2}+0.1\right)}{0.02}, \rho_{4}(\theta(t))=\frac{\bar{\theta}_{1}-\theta_{1}}{\bar{\theta}_{1}-\underline{\theta}_{1}} \frac{\bar{\theta}_{2}-\theta_{2}}{\bar{\theta}_{2}-\underline{\theta}_{2}}=\frac{\left(0.05-\theta_{1}\right)\left(0.1-\theta_{2}\right)}{0.02}
\end{aligned}
$$

\subsection{UIPIO Design}

The polytopic Unknown Input Proportional Integral Observer is represented by

$$
\left\{\begin{array}{l}
\dot{z}(t)=\sum_{i=1}^{4} \rho_{i}(\theta(t))\left(N_{i} z(t)+G_{i} u(t)+L_{i} y(t)+T_{i} \hat{d}(t)\right) \\
\hat{x}(t)=z(t)+H_{2} y(t) \\
\dot{\hat{d}}(t)=\sum_{i=1}^{4} \rho_{i}(\theta(t)) \Phi_{i}(y(t)-\hat{y}(t))
\end{array}\right.
$$

The design procedure of the polytopic UIPIO is thus given as follows:

(i) Based on the system matrices, assumptions $A_{2}$ and $A_{3}$ are satisfied.

(ii) Matrices $H_{1}$ and $H_{2}$ are obtained from equation (26).

(iii) By using (21), LMI (47) can be efficiently solved via numerical approach using the LMI toolbox to determine the gains matrices. Therefore, these inequalities are fulfilled with:

$$
\begin{aligned}
K_{1} & =\left[\begin{array}{ccc}
-1.1672 & 2.7989 & -2.2082 \\
5.1350 & -5.8683 & 6.9783 \\
3.9170 & 5.6084 & -5.1706 \\
-8.4141 & 9.8486 & 0.1051
\end{array}\right], K_{2}=\left[\begin{array}{ccc}
-1.1179 & 2.7162 & -2.1058 \\
5.0955 & -5.6972 & 6.7604 \\
2.7157 & 6.7906 & -5.9916 \\
-7.6181 & 8.8765 & 0.8780
\end{array}\right] \\
K_{3} & =\left[\begin{array}{ccc}
-1.2822 & 2.9205 & -2.3191 \\
5.5822 & -6.3372 & 7.3882 \\
4.2007 & 5.3086 & -4.9580 \\
-8.8752 & 10.3629 & -0.3293
\end{array}\right], K_{4}=\left[\begin{array}{ccc}
-1.2307 & 2.8367 & -2.2169 \\
5.5333 & -6.1608 & 7.1708 \\
2.9920 & 6.4925 & -5.7753 \\
-8.0687 & 9.3878 & 0.4393
\end{array}\right] \\
\Phi_{1} & =\left[\begin{array}{lll}
38.2002 & -37.1450 & 31.6370
\end{array}\right], \Phi_{2}=\left[\begin{array}{ccc}
37.0113 & -35.2044 & 29.8070
\end{array}\right] \\
\Phi_{3} & =\left[\begin{array}{llll}
42.2863 & -41.4254 & 35.1279
\end{array}\right], \Phi_{4}=\left[\begin{array}{lll}
41.0013 & -39.4316 & 33.3043
\end{array}\right]
\end{aligned}
$$

where $K_{i}$ are the proportional and $\Phi_{i}$ are the integral gains matrices.

(iv) The remaining polytopic UIPIO matrices $G_{i}, N_{i}$ and $L_{i}$ can be established by (19), (28) and (29) respectively.

The input signals are constant: $u_{1}(t)=u_{2}(t)=2$, the disturbance $d(t)$ is a rectangular signal applied for $7 \leq t \leq 10$. The initial values of the original states $x(t)$ and the unknown input $d(t)$ are equal to 0 and the initial values of the estimated states are: $\hat{x}(t)=\left[\begin{array}{llll}0.5 & 1 & 0.5 & -0.5\end{array}\right]^{T}$. The state estimation and unknown inputs estimation given by the polytopic UIPIO are shown on the following figures. 

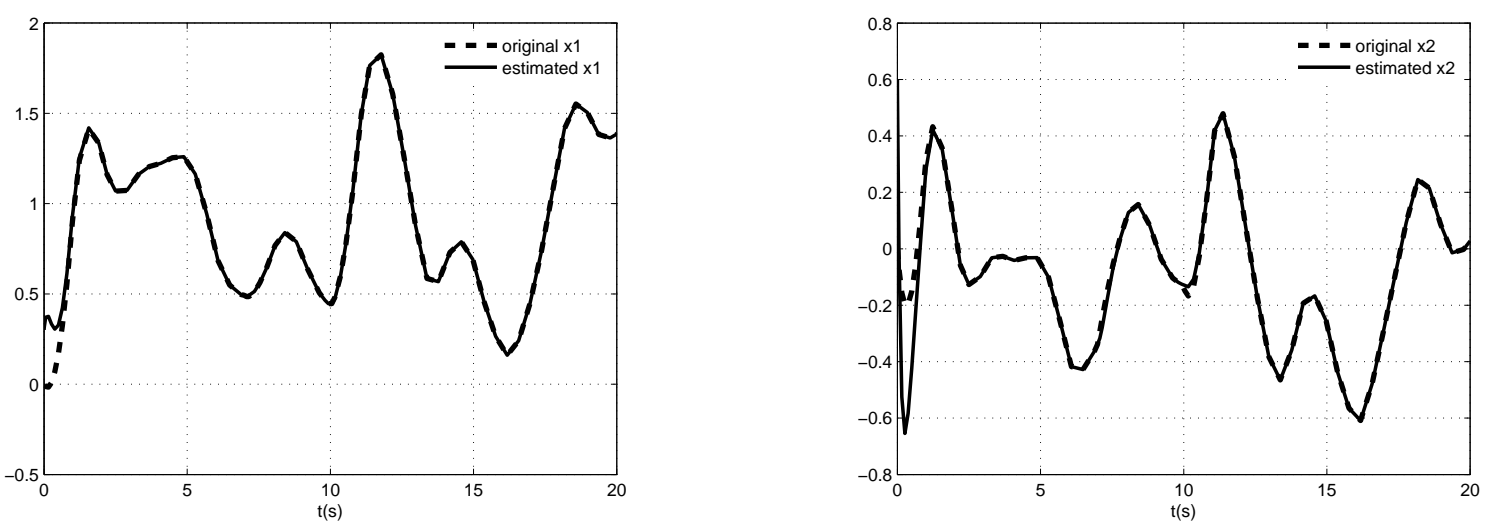

Figure 1. Original state $x_{1}(t)$ and its estimated $\hat{x}_{1}(t)$

Figure 2. Original state $x_{2}(t)$ and its estimated $\hat{x}_{2}(t)$
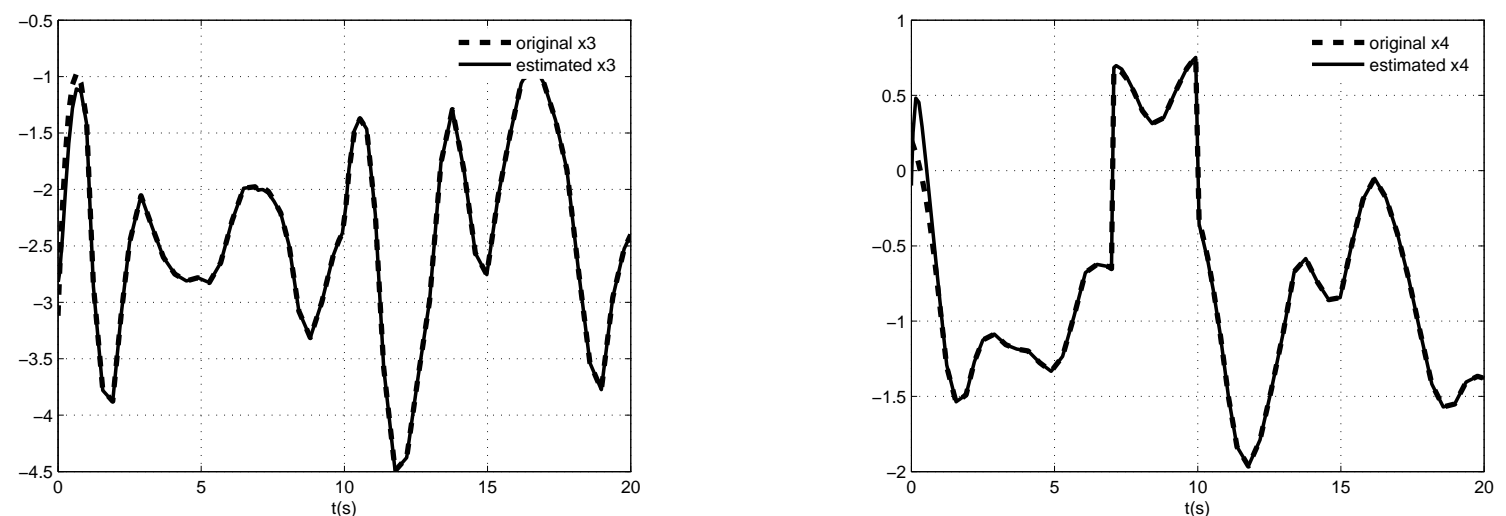

Figure 3. Original state $x_{3}(t)$ and its estimated $\hat{x}_{3}(t)$

Figure 4. Original state $x_{4}(t)$ and its estimated $\hat{x}_{4}(t)$

The behavior of the polytopic UIPIO is shown on figures (1) to (4). It can be observed that the estimated states can closely track the original states of the polytopic descriptor system affected by an unknown input in spite of measurements which are perturbed by a white Gaussian noise with variance 0.01 .

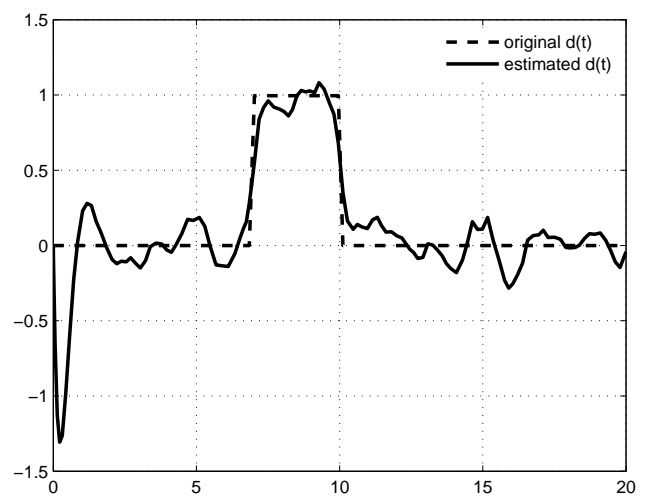

Figure 5. Unknown input $d(t)$ and its estimated $\hat{d}(t)$ 
In addition, as seen on the figure (5), the polytopic UIPIO provides a correct unknown input estimation in spite of noise and disturbances. The polytopic UIPIO design is easy but the restriction for this polytopic UIPIO is to consider that disturbances have slow variation i.e. $\dot{d}(t) \simeq 0$. However, in practice, we can relax this constraint.

\subsection{Fault diagnosis with polytopic UIPIO}

In this paragraph, we will only consider a constant matrix $B$ and two actuator faults with constant magnitudes. Let us consider the model (61) and the polytopic UIPIO (49) with the same previous matrices except for a constant matrix $B(\theta)$ :

$B(\theta)=B=\left[\begin{array}{cccc}1 & 1 & 1 & 0 \\ 1 & 0.5 & 0 & 0\end{array}\right]^{T}$ and $R(\theta)=R=\left[\begin{array}{llll}0 & 0.6 & 0 & 1\end{array}\right]^{T}$.

In this part, we recall that $F^{j}$ is the $j^{t h}$ column of $B$ and $F^{r}$ is the matrix $B$ without the $j^{t h}$ column. In order to detect and isolate the actuators faults, two polytopic UIPIO are designed here, each one designed to isolate an actuator fault. Let consider $f_{1}(t)$ and $f_{2}(t)$, the fault signals which represent control input dysfunctions of $u_{1}(t)$ and $u_{2}(t)$ respectively and defined by:

$$
f_{1}(t)=\left\{\begin{array}{l}
0.25 u_{1}(t) \text { for } 10 \leq t \leq 14 \\
0 \quad \text { elsewhere }
\end{array} \text { and } f_{2}(t)=\left\{\begin{array}{c}
0.4 u_{2}(t) \text { for } 15 \leq t \leq 19 \\
0 \quad \text { elsewhere }
\end{array}\right.\right.
$$

The first polytopic UIPIO is designed to estimate and isolate the first actuator fault $f_{1}(t)$ and a second one is also designed to estimate and isolate the second actuator fault $f_{2}(t)$. The performance of the fault diagnosis system is illustrated for two actuator faults as shown below.

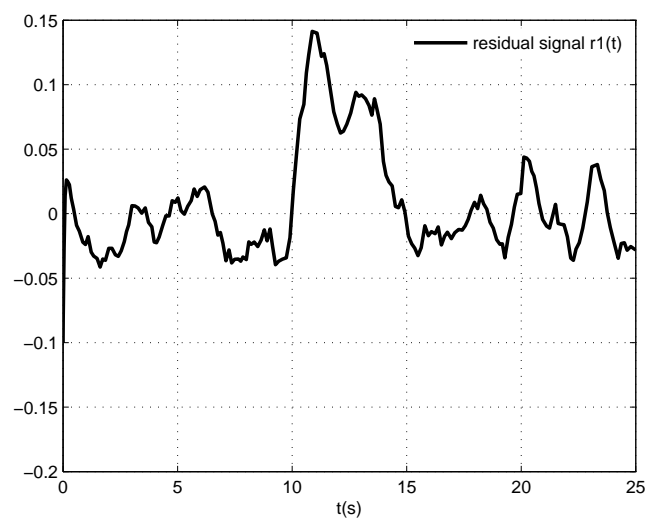

Figure 6. the residual signal $r_{1}(t)$

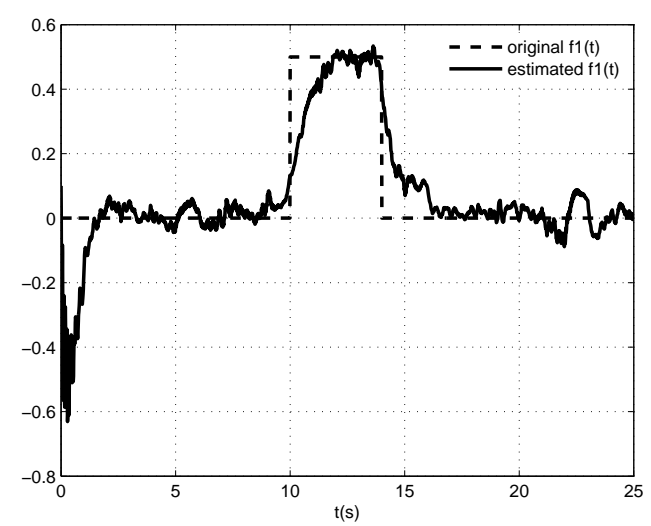

Figure 8. first actuator fault $f_{1}(t)$ and its estimated $\hat{f}_{1}(t)$

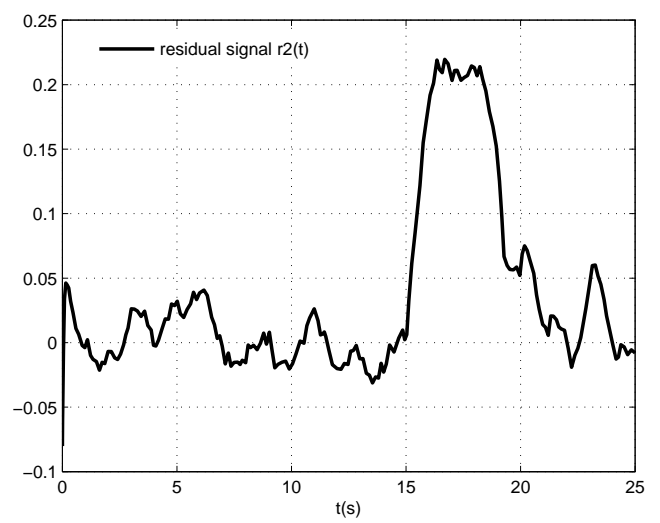

Figure 7. the residual signal $r_{2}(t)$

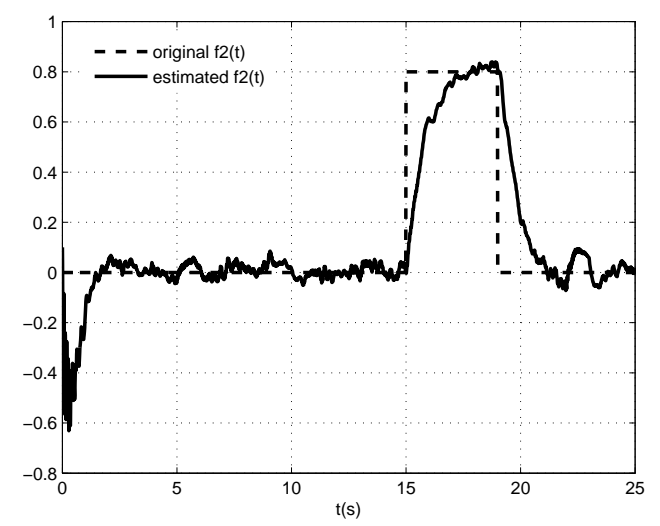

Figure 9. second actuator fault $f_{2}(t)$ and its estimated $\hat{f}_{2}(t)$ 
Figures (6) and (7) display the isolation task. One can see that the residual is almost zero throughout the time simulation run for fault-free residuals despite noisy environment and increase in magnitude considerably when actuator faults occur at time $t=10 \mathrm{~s}$ (Fig.(6)) and at time $t=15 \mathrm{~s}$ (Fig.(7)). Then, faults are easily isolated using the information provided by residuals signals.

Figures (8) and (9) display the estimation of $f_{1}(t)$ and $f_{2}(t)$. An error appears in the estimation of both $f_{1}(t)$ and $f_{2}(t)$ respectively. This is due to the fact that when those faults stop abruptly, there is a remaining time to give an estimation of $f_{1}(t)$ and $f_{2}(t)$ owing to the response of this polytopic UIPIO. It is important to note that without noise, the estimation is better but we want to present a more realistic point of view in a noisy environment.

\section{CONCLUSION}

This paper has addressed the problem of state estimation, fault detection and isolation for dynamic systems that can be described by polytopic LPV descriptor models subject to disturbances. The state and unknown inputs estimation are obtained by a polytopic Unknown Inputs Proportional Integral Observer which has been used here for the detection, isolation and estimation of actuators faults in spite of disturbances. Stability conditions of this polytopic UIPIO have been formulated and solved within a linear matrix inequality framework. The developed FDI scheme for LPV descriptor systems has been applied into an example in order to estimate both state vector and disturbances. Based on fault estimation, a fault tolerant control scheme for LPV descriptor systems could be synthesized so as to enhance robustness of the system.

\section{REFERENCES}

1. AI-Guo Wu and Guang-Ren Duan. Generalized PI observer design for linear systems. IMA Journal of Mathematical Control and Information 2008, vol 25, pp 239-250.

2. Anstett F. and Millerioux G. and Bloch G. Polytopic observer design for LPV systems based on minimal convex polytope finding Journal of Algorithms and Computational Technology, vol 3, N1, 2009, pp 23-43.

3. Bokor J. and Szabo Z. Fault detection and isolation in nonlinear systems Annual Reviews in Control., vol 33, N 2, 2009, pp 113-123.

4. Boutayeb M. and Darouach M. Observers design for non linear descriptor systems Proceedings of the 34th Conference on Decision and Control, New Orleans, 1995, pp 2369-2374.

5. Boyd S.and El Ghaoui L. and Feron E. and Balakrishnan V. Linear Matrix Inequalities in System and Control Theory SIAM, Studies In Applied Mathematics.

6. Bruzelius F. Linear Parameter-Varying Systems an approach to gain scheduling PhD Thesis, Department of Signals and Systems, Chalmers University of Technology, Goteborg, Sweden.

7. Chen J. and Patton R.J. Robust model-based fault diagnosis for dynamic systems Kluwer Academic Publishers, 1999.

8. Darouach M. and Boutayeb M. Design of Observers for Descriptor Systems IEEE transactions on Automatic Control, vol 40, 1995, pp 1323-1327.

9. Grenaille S., Henry D. and Zolghadri A. A method for designing FDI filters for polytopic LPV models. safeprocess, 2006.

10. Hamdi H., Rodrigues M., Mechmeche C., Theilliol D. and BenHadj Braiek N., State Estimation for Polytopic LPV Descriptor Systems: Application to Fault Diagnosis. the 7th IFAC Symposium on Fault Detection, Supervision and Safety of Technical Processes, Barcelona, Spain, June 30 - July 3, 2009 pp 438-443.

11. Hou M. and Muller P. C. Observer Design for Descriptor Systems. IEEE Transactions on Automatic Control 1999, Vol 44, pp 164-168.

12. Koenig D. and Mammar S. Design of Proportional-Integral Observer for Unknown Input Descriptor Systems. IEEE transactions on automatic control, december 2002, vol 47, N12.

13. Koenig D. Unknown Input Proportional Multiple-Integral Observer Design for Linear Descriptor Systems: Application to State and Fault Estimation. IEEE Transactions on Automatic control February 2005; Vol 50, N2, pp 212-217.

14. Marx B., Koenig D. and Ragot J. Design of observers for Takagi-Sugeno descriptor systems with unknown inputs and application to fault diagnosis IET Control Theory Appl., vol N1, 2007, pp 1487-1495.

15. Mason P. and Sigalotti M. and Daafouz J. On stability analysis of lilnear discrete-time switched systems using quadratic Lyapunov Functions Proceedings of 46th IEEE Conference Decision and Control, New Orleans, LA 2007, pp 5629-5633.

16. Masubuchi I., Kato J., Saeki M. and Ohara A., Gain-Scheduled Controller Design Based on Descriptor Representation of LPV Systems: Application to Flight Vehicle Control. 43rd IEEE Conference on Decision and Control, Atlantis, Paradise Island, Bahamas, December 14-17, 2004, pp 815-820. 
17. Masubuchi I. and Suzuki A., Gain-Scheduled Controller Synthesis Based on New LMIs for Dissipativity of Descriptor LPV Systems The International Federation of Automatic Control, Proceedings of the $17^{\text {th }}$ World Congress, Seoul, Korea, July 6-11, 2008, pp 9993-9998.

18. Orjuela R., Marx B., Maquin D. and Ragot J., State estimation for nonlinear systems using decoupled multiple model. International Journal of Modelling, Identification and Control 2008, Vol. 4(1), pp. 59-67

19. Rodrigues M., Theilliol D., Adam-Medina M., and Sauter D., A Fault Detection and Isolation Scheme for Industrial Systems based on Multiple Operating Models. Control Engineering Practice 2008, Vol N16, pp 225-239.

20. Rodrigues M., Theilliol D., Aberkane S. and Sauter D. Fault Tolerant Control Design for Polytopic LPV Systems. International Journal of Applied Mathematics and Computer Sciences, 2007, Vol N17, pp 27-37.

21. Rodrigues M., Theilliol D. and Sauter D. Design of a Robust Polytopic Unknown Input Observer for FDI: Application to Nonlinear Systems described by a Multi-Models Representation. 44th IEEE Conference on Decision and Control and European Control, Conference ECC, Sevilla, Spain 2005, pp 6268-6273.

22. Taniguchi T., Tanaka K., and Wang H. O. Fuzzy Descriptor Systems and Nonlinear Model Following Control. IEEE Transactions on Fuzzy Systems, 2000, Vol N 8, pp 442-452.

23. Theilliol D., Noura H. and Ponsart J.C. Fault diagnosis and accommodation of three-tank system based on analytical redundancy. ISA Transactions, 2002, Vol N 41, pp 365-382.

24. Wang Y., Sun Z. Q. and Sun F. C. Robust Fuzzy Control of a Class of Nonlinear Descriptor Systems with TimeVarying Delay. International Journal of Control, Automation and Systems, 2004, Vol N 2, pp 76-82.

25. Wu F. Control of Parameter Varying Systems PhD Thesis, University of california at berkeley, USA.

26. Zolghadri A., Henry D. and S. Grenaille Fault diagnosis for LPV systems. 16th Mediterranean Conference on Control and Automation, MED'08, Ajaccio, 2008, 